\title{
Validation of In Vivo Patellar Tracking with a Custom-Made Clamp: A Fluoroscopy Study
}

\author{
Chen-Yi Song ${ }^{1}$, Jyh-Horng Wang ${ }^{2}$, Wei-Chun Chen ${ }^{1}$, Mei-Hwa Jan ${ }^{3}$ and Jiu-Jenq Lin ${ }^{*}, 1$ \\ ${ }^{I}$ School and Graduate Institute of Physical Therapy, College of Medicine, National Taiwan University, Taipei, Taiwan \\ ${ }^{2}$ Department of Orthopaedic Surgery, National Taiwan University Hospital, Taipei, Taiwan \\ ${ }^{3}$ Yeong-An Clinic, Taipei, Taiwan
}

\begin{abstract}
Evaluation of patellar tracking has been suggested as an increasingly relevant diagnostic tool in patients with patellofemoral disorders. This study aimed to assess the accuracy of measuring in vivo patellar tracking with the use of a custom-made clamp. Nine healthy female college students participated. Sagittal-view fluoroscopy was used to track the movement of the clamp and the underlying patella of each subject's dominant leg during a lunge. The movement patterns (tilt angle and displacement) as well as the absolute angle/distance between the patella and clamp were investigated at 7 knee flexion angles $\left(0^{\circ}, 15^{\circ}, 30^{\circ}, 45^{\circ}, 60^{\circ}, 75^{\circ}\right.$, and $\left.90^{\circ}\right)$. The patella and clamp showed similar movement patterns (tilt angle: $r=0.94, P=0.002$; displacement: $r=0.90, P=0.007$ ). The root mean squared error of less than $3.5^{\circ}$ and $0.6 \mathrm{~cm}$ were identified below knee flexion $60^{\circ}$. The absolute angle significantly changed at knee flexion $90^{\circ}$ compared to $0^{\circ}(P=$ 0.016). The absolute distance between the upper edge of the patella and clamp significantly changed at knee flexion $60^{\circ}$ to $90^{\circ}$ as compared to $0^{\circ}$ (all $P<0.05$ ). The angle/distance change may represent an influence of a skin motion artifact manifesting beyond knee flexion $60^{\circ}$. With careful consideration, the custom-made clamp may offer reasonably accurate representations of patellar motion that can be used to further study patella pathologies and develop computational models.
\end{abstract}

Keywords: Patella, patellar tracking, knee, patellofemoral, patellofemoral pain, fluoroscopy.

\section{INTRODUCTION}

Patellofemoral pain syndrome (PFPS) is a very common knee disorder that occurs in young and physically active people. It affects about $25 \%$ of the total population [1]. Among them, females are more frequent pain suffers [2]. Patients often complain of anterior or retropatellar pain typically produced in squatting, kneeling, ascending or descending stairs, running, and jumping, or after prolonged sitting. The mechanism of PFPS is considered to be multifactorial [3]. One of the possible, and most commonly accepted, causes in the clinical community is lateral malalignment or maltracking (defined as abnormality of static position or dynamic orientation) of the patella on the femur during knee flexion and extension [4]. This lateral patellar migration potentially puts uneven stresses on both the patella itself and the peripatellar tissues, leading to PFPS $[4,5]$.

Study of patellofemoral kinematics is gaining much attention in the research field of PFPS. To study the patellar tracking patterns in vivo quantitatively and noninvasively, several imaging techniques, such as X-ray, computerized tomography (CT), and both dynamic and static magnetic resonance imaging (MRI), are frequently used clinically [610]. However, these researchers typically focused on 2D alignment, and only one study, to our knowledge, has

*Address correspondence to this author at the Taiwan/School and Graduate Institute of Physical Therapy, College of Medicine, National Taiwan University, Floor 3, No.17, Xuzhou Rd., Zhongzheng District, Taipei City 100, Taiwan; Tel: 886-2-33668126; Fax: 886-2-33668161;

E-mails: jiujlin@ntu.edu.tw,d96428002@ntu.edu.tw quantified 3D patellofemoral and tibiofemoral kinematics using MRI [10]. Recent studies have also investigated patellar tracking in weight-bearing knee flexion using durafluoroscopes and MRI $[11,12]$. This technique required 3D models to reproduce the in vivo knee motion, which might present a technical challenge for general clinical application. Investigation with a motion system, on the other hand, can obtain the patellar tracking data in weight-bearing knee function [13, 14], which is more closely related to daily activity. Nevertheless, the skin motion artifact, which is the displacement between the marker and underlying bone, is a serious source of error that would interfere with measurement accuracy in the motion analysis. As the patella is the biggest sesamoid bone in the human body, several research methods, such as the custom-made clamp method, have been developed as consequence for in vivo and noninvasive patellar tracking measurement to track patella movement during functional activities.

Three studies have used patellar clamps for real-time evaluation of patellar tracking with a motion system [13-15]. Lin et al. [15] corroborated the patellar tracking based on the patellar clamp using anterior and medial views of fluoroscopic imaging with subjects' knee flexion-extension in the range of $20^{\circ}$. The authors recorded the imaging with videotape, and only the visual inspection method was used to compare the patella and patellar clamp movements. They concluded that the patellar clamp closely followed the patellar movement during voluntary knee motion within $20^{\circ}$ of knee motion. Laprade and Lee [13] later examined the patella and patellar clamp during knee flexion (squat) up to $90^{\circ}$. They found that the displacement of the patellar mould 
was able to truly reflect that of the patella up to $60^{\circ}$ of knee flexion, where the difference in the displacement of the patella and mould along the long axis of femur was found to be less than $3 \mathrm{~mm}$. Wilson et al. [14] calculated the general displacement of the patella and clamp, and reported that the mean difference was $0.97 \mathrm{~mm}$ (range: $0-2.43 \mathrm{~mm}$ ) during knee flexion up to approximately $105^{\circ}$ from the sagittalplane fluoroscopic image. Although these authors used fluoroscopic imaging to qualitatively or quantitatively validate the in-vivo patellar tracking method with the use of a custom clamp, these reports were collateral results accompanying their main research. The number of subjects was limited, and only the displacement data were generated $[13,14]$.

The validity of a measurement tool is important for pathology documentation and outcome assessments in both the clinic and research. Since evidence regarding the accuracy of patellar tracking with the use of custom clamps is still unclear, the purpose of this study was to validate an in vivo patellar tracking method with the use of a custom clamp. We hypothesized that (1) the skin motion artifact would be augmented with knee flexion, thereby decreasing the consistency of the movement pattern (tilt angle and displacement) between the patella and clamp; and (2) the absolute angle/distance difference between the patella and clamp would change with degree of knee flexion. In addition, the reliability of the fluoroscopic imaging measurements was also investigated.

\section{MATERIALS AND METHODOLOGY}

\section{Subjects}

Nine healthy female college students (mean age: 21.2 (SD 1.6) yr, mean height: 161.9 (SD 8.0) cm, mean weight: 53.2 (SD 7.8) kg) participated. All of them were right-leg dominant. Subjects were excluded if they had: (1) previous significant knee, hip, or ankle pathology, traumatic history or surgery; or (2) current or past symptoms of patellofemoral pain. All participants signed an informed consent statement approved by the ethics committee of the National Taiwan University Hospital prior to taking part in this study.

\section{Measurement of Patellar Tracking by Fluoroscopy}

A custom-made patellar clamp was created using Thermoplast $^{\mathrm{TM}}$ material and the methods described by Laprade and Lee [13]. The Thermoplast was first heated to make it malleable enough to conform closely to the patella. The subject was seated with the knee slightly flexed. The edges of the clamp were pressed firmly around the perimeter of the patella, and in particular, the medial and lateral sides were curved inward on the edges of the patella. The clamp was then affixed to the skin overlying the center of the patella using double-sided tape. A radio-opaque marker was attached onto the center of the clamp (Fig. 1). Sagittal-view fluoroscopy (BV Endura; Philips Inc., Eindhoven, Netherlands) was used to examine the movement of the marker and the underlying patella of each subject's dominant leg during a lunge of up to $90^{\circ}$ (Fig. 1). During the lunge, the subjects were positioned with their dominant knee in the field of view of the fluoroscopy. The subject was first requested to stand upright with the knee straight while data were collected for a neutral position $\left(0^{\circ}\right.$ of knee flexion).
This data was used as the reference position for determining the relative positional change of the clamp (marker) and patella. Subjects then performed 6 lunges with their testing knee bent to $15^{\circ}, 30^{\circ}, 45^{\circ}, 60^{\circ}, 75^{\circ}$, and $90^{\circ}$ of knee flexion, monitored by goniometry, in a random sequence. Subjects were instructed to support their weight on the leg being studied, and they were also allowed to use the contralateral leg for weight support and balance. At each joint angle, subjects held the position for a brief moment, and the fluoroscopic image was recorded.

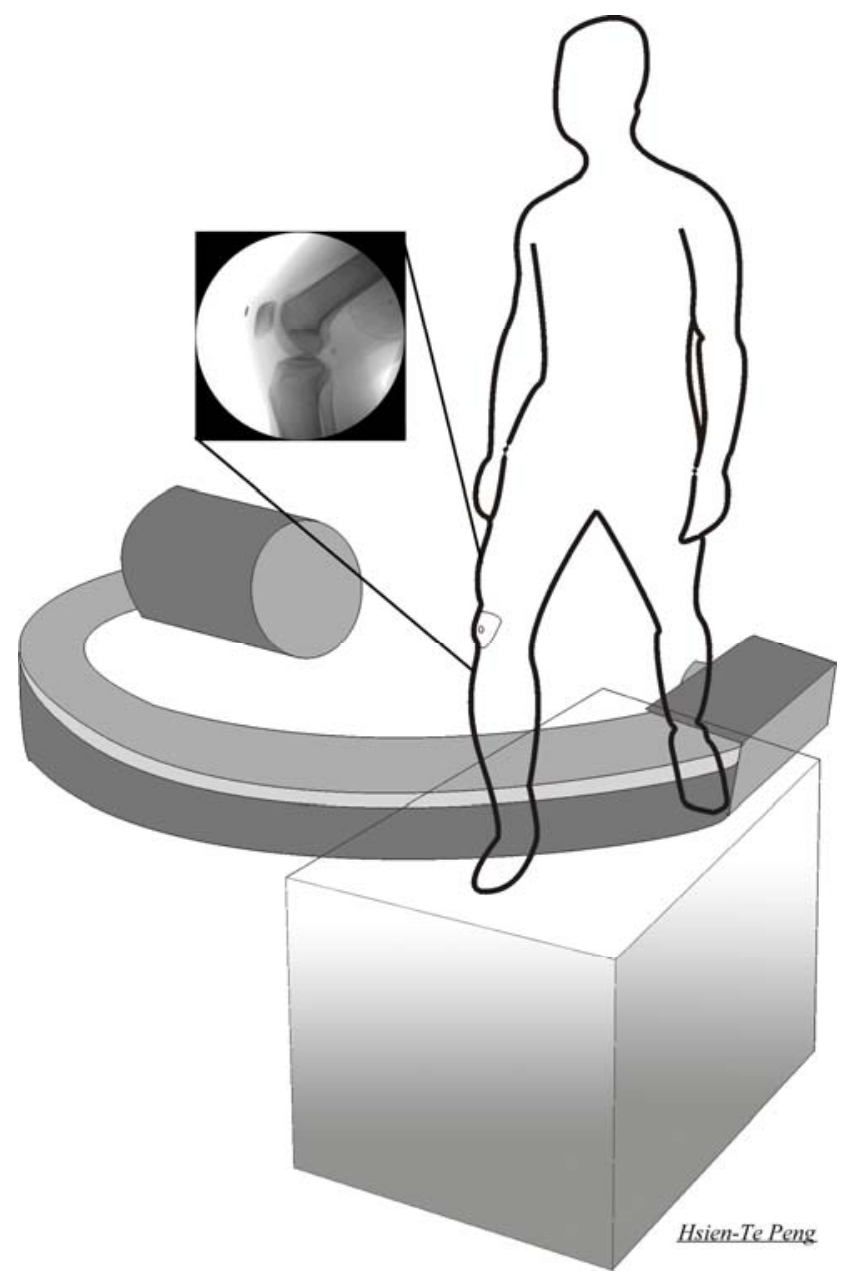

Fig. (1). Lunge in fluoroscopic image system.

\section{Data Analysis}

The fluoroscopic images were analyzed by 2 blinded assessors. The movement patterns (tilt angle and displacement) as well as the absolute angle/distance between the patella and clamp (marker) were investigated at 7 knee flexion angles $\left(0^{\circ}, 15^{\circ}, 30^{\circ}, 45^{\circ}, 60^{\circ}, 75^{\circ}\right.$, and $\left.90^{\circ}\right)$. The tilt angle of patella (Tp)/clamp (Tc) was defined as the angle subtended by the horizontal line and the diagonal line (connection of upper and lower edges of the patella/marker) (Fig. 2). The displacement of patella (Dp)/clamp (Dc) was defined as the distance between the tibial tuberosity to the lower edge of the patella/marker (Fig. 2). The absolute angle was defined as the angle between the diagonal lines of the patella and marker. The absolute distance was between the upper or lower edge of the patella and marker. These parameters were digitized and measured using Sante 
DICOM viewer 1.4.7 software (Santesoft Inc., Athens, Greece).

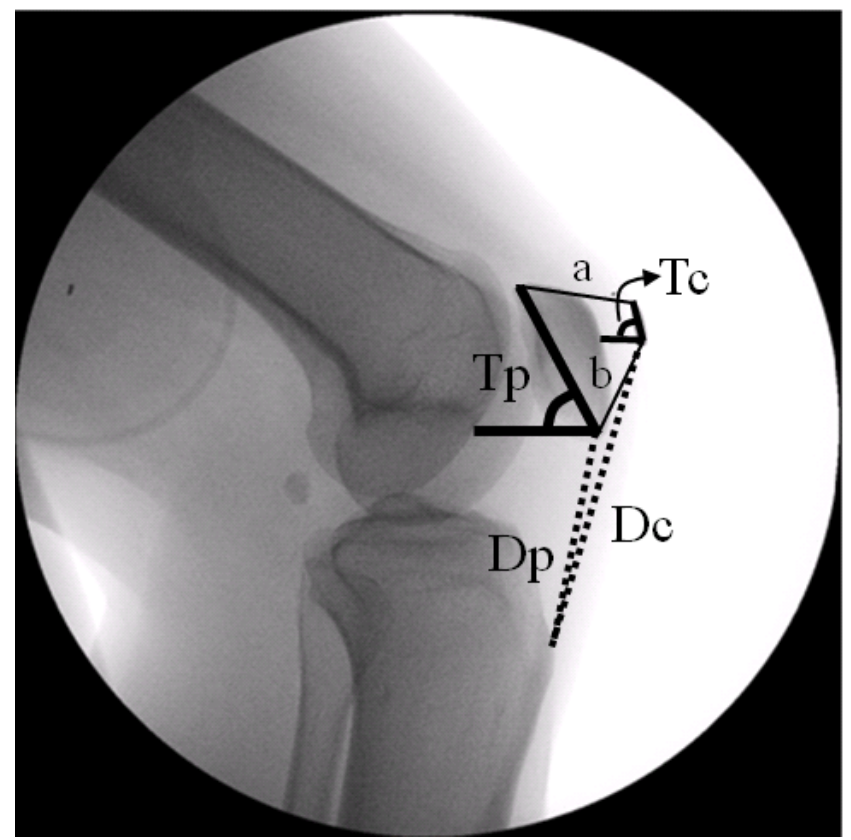

Fig. (2). Tilt angle of patella (Tp)/clamp (Tc) and displacement of patella (Dp)/clamp (Dc).

\section{Statistical Analysis}

Data were analyzed using SPSS 13 software (SPSS Inc., Chicago, IL). For the movement pattern, the tilt angle and displacement of the patella/clamp were compared by calculating the Pearson correlation coefficient $(r)$ and root mean squared error (RMSE) between the two curves (patella vs clamp). The RMSE can be thought of as a global estimator of the similarity relation, and this parameter is optimal when it is equal to 0 [16].

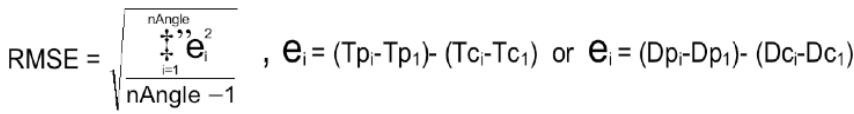

For the absolute angle/distance between the patella and clamp, the one-way repeated ANOVA at 7 knee flexion angles were calculated twice at a significant alpha level of 0.05 , with Bonferroni follow-up analyses for multiple pairwise comparisons where appropriate. The intraclass correlation coefficient (ICC) was used to determine the intertester reliability of the fluoroscopic imaging measurements. An ICC of greater than 0.75 is considered good reproducibility [17].

\section{RESULTS}

The patella and clamp showed similarities in movement pattern. Both tilt and displacement patterns were highly correlated (tilt angle: $r=0.94, P=0.002$; displacement: $r=0.90, P=0.007$ ) (Fig. 3A, B). The RMSE for tilt angle was found to be less than $3.5^{\circ}\left(1.0^{\circ}\right.$ to $\left.3.5^{\circ}\right)$ at knee flexion of less than $60^{\circ}$, and became large $\left(6.3^{\circ}\right.$ to $\left.7.6^{\circ}\right)$ beyond knee flexion of $60^{\circ}$. The RMSE for displacement became gradually larger from $0.24 \mathrm{~cm}$ at knee flexion of $15^{\circ}$ to 0.83 $\mathrm{cm}$ at knee flexion of $90^{\circ}$ (a RMSE of $0.53 \mathrm{~cm}$ on average).
The absolute angle significantly changed at knee flexion of $90^{\circ}$ compared to that at knee flexion of $0^{\circ}(P=0.016)$ (Fig. 4A). The absolute distance between upper edge of the patella and marker significantly changed at knee flexion of $60^{\circ}$ $(P=0.041), 75^{\circ}(P=0.022)$ and $90^{\circ}(P=0.004)$ compared to that at knee flexion of $0^{\circ}$ (Fig. 4B).

Good reliability was shown in all fluoroscopic imaging measurements. The mean ICC for the patellar tilt was 0.92 (range: 0.87-0.99 across all knee flexion angles), and the corresponding value for the clamp tilt was 0.92 (range: $0.86-$ 0.98 ). For displacement pattern measurement, the mean ICC values were 0.85 (range: $0.82-0.89$ ) and 0.91 (range: 0.88 0.93 ) for the patella and clamp, respectively. The mean ICC was 0.85 (range: $0.76-0.93$ ) for the absolute angle, 0.86 (range: 0.80-0.91) and 0.96 (range: 0.93-0.99) for the absolute distance between upper and lower edges of the patella and clamp.

\section{DISCUSSION}

The aim of the current study was to validate an in vivo patellar tracking method with the use of a custom clamp that could easily be included in clinical testing conditions. The movement patterns (tilt angle and displacement) as well as the absolute angle/distance between the patella and clamp (marker) were investigated during lunges of up to $90^{\circ}$ in knee flexion increments of $15^{\circ}$. Similarities in movement pattern were found between patella and clamp. However, the findings in the RMSE for tilt angle and displacement gradually became larger with knee flexion. Less than $3.5^{\circ}$ and $0.6 \mathrm{~cm}$ error (RMSE) were identified below knee flexion of $60^{\circ}$. The angle/distance change between the patella and clamp may represent an influence of skin motion artifact at knee flexion of $75^{\circ}$ and $90^{\circ}$. The distance between the upper edge of the patella and marker was found to significantly decrease, while the lower edge also demonstrated a trend of increasing distance, without reaching statistical significance, at the deepest knee flexion angle $(P=0.069)$. This means that as the patella downwardly enters and seats itself into the trochlea groove with gradual knee flexion, the clamp can no longer firmly grip the perimeter of the patella, which causes upward movement of the marker relative to the patella, and subsequently changes the angular relationship between the patella and clamp. The results support the hypothesis that error was augmented with knee flexion, resulting in inconsistency of the movement pattern between the patella and clamp. The movement pattern inconsistency responded to knee flexion angles beyond $60^{\circ}$.

The current study obtained a good intertester reliability on the fluoroscopic imaging measurements, since precise landmark criteria were used in all quantitative measures. Previous studies reported that the general difference in the displacement of the patella and clamp was less than $3 \mathrm{~mm}$ $[13,14]$. As we transformed our data into a comparable form, the mean difference between the patella and clamp relative to tibial tuberosity was $2.2 \mathrm{~mm}$ (range: $1.0-3.0 \mathrm{~mm}$ ) within a $90^{\circ}$ lunge, which was similar with a previous report of the mean difference of $0.97 \mathrm{~mm}$ (range: $0-2.43 \mathrm{~mm}$ ) during knee flexion of up to approximately $105^{\circ}$ [14]. Our findings are also in agreement with, and to some degree better than, those of Laprade and Lee [13], who found that 


\section{A. Tilt angle}

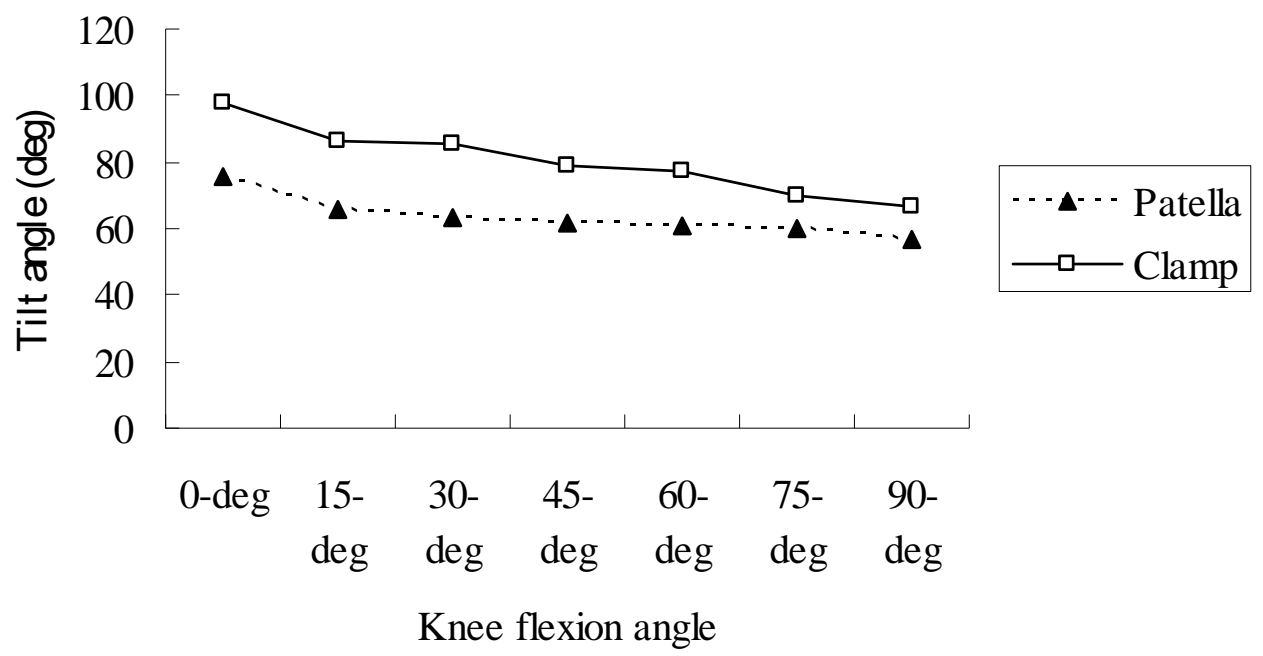

\section{B. Displacement}

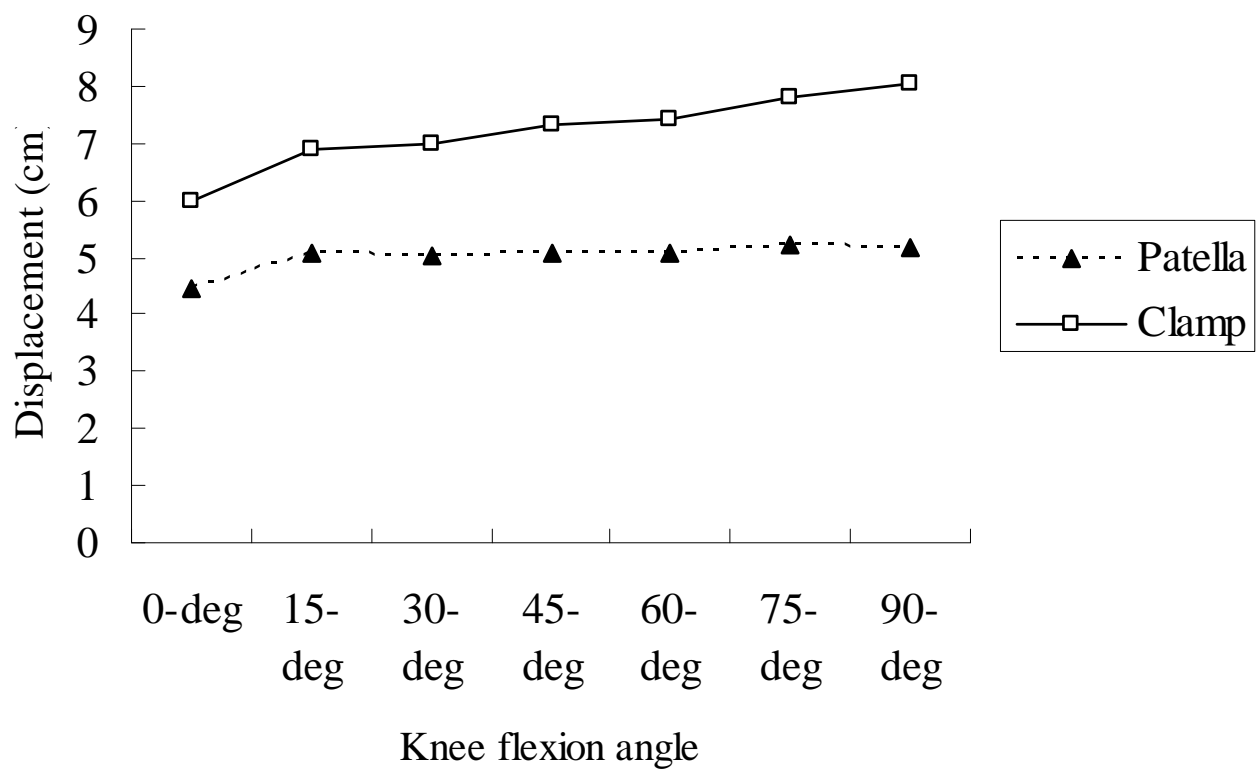

Fig. (3). Movement patterns (A. tilt angle and B. displacement) of the patella and clamp during lunge.

the difference in the displacement of the patella and clamp along the long axis of the femur was less than $3 \mathrm{~mm}$ for knee flexion of less than $60^{\circ}$, and became unacceptably large beyond that angle. We suggest that the mastery and technique of the skilled individual who makes such a customized clamp may influence the results. Developing standardized procedures for making the clamp and training users to meet a criterion-based level of competence would likely improve the reliability and validity of the customized clamp approach.
This is the first study, to our knowledge, to investigate the angular relationship between the patella and clamp. The mean difference between the patella and clamp angle relative to horizontal was $2.0^{\circ}$ (range: $0.1-6.8^{\circ}$ ) within a $90^{\circ}$ lunge. The maximal difference $\left(6.8^{\circ}\right)$ occurred at $75^{\circ}$ of knee flexion. The absolute angle change and displacement between the patella and clamp, manifested beyond knee flexion of $60^{\circ}$, would decrease the measurement accuracy of in vivo patellar tracking. Since evaluation of patellar tracking has been suggested as an increasingly relevant diagnostic tool in patients with patellofemoral disorders, the custom- 


\section{A. Absolute angle}

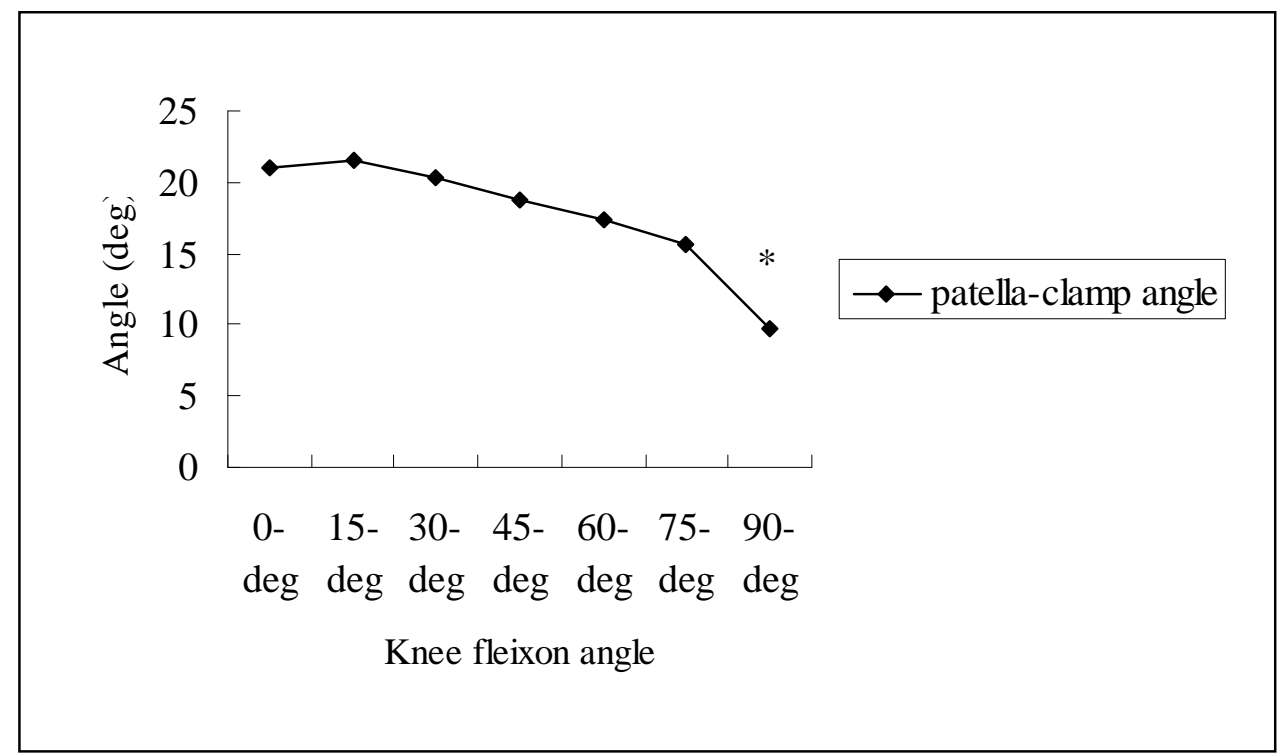

\section{B. Absolute distance}

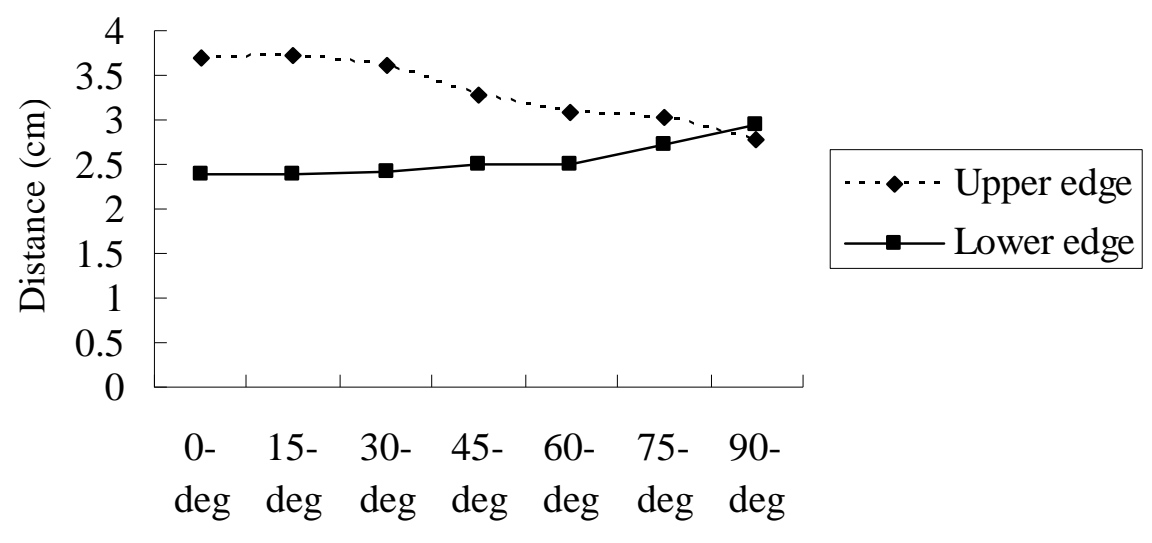

Knee flexion angle

Fig. (4). A. Absolute angle between the patella and clamp; B. Absolute distance between upper and lower edge of the patella and clamp (marker) during lunge movement. ${ }^{*} \mathrm{P}<0.05$.

made clamp, even if not optimal, may offer reasonably accurate representations of patellar motion. For clinical application, the current study results could serve as a validity basis for further study of patella pathologies and the development of computational models. Better knowledge of patellar tracking will provide appropriate guidelines for planning physiotherapeutic programs thereafter.

The limitations of our study should be noted. Only sagittal-view fluoroscopy was used in this study. The alterations of patellar tracking associated with patellofemoral pain were not limited to the axial plane (lateral displacement and tilt) [10]. Given that the patellar flexion/extension and superior/inferior displacement have larger movements than the other two components of patellar motion (spin, tilt, anterior/posterior, and medial/lateral displacement) during knee movement $[10,14]$, the patella and clamp motion artifact on the other planes is expected to be less than what we discovered in this study. However, future study is needed to address this speculation. Additional work is also needed to solve the problem associated with motion artifacts using the patellar clamp method or other methods to track patellar motion, especially with a large range of knee motion. 


\section{CONCLUSIONS}

We have presented a comprehensive analysis of the accuracy of custom made clamp method for collecting patella tracking in vivo. With careful consideration, the custom-made clamp may offer reasonably accurate representations of patellar motion that can be used to further study patella pathologies and develop computational models. Although the movement pattern consistency and absolute angle/distance errors were found to be reasonable, the usefulness of this method may depend on specific experimental conditions. This method has applications in tracking patella motions with patellofemoral pathology, as well as assessing changes in patellar tracking after treatment intervention.

\section{ACKNOWLEDGEMENTS}

We thank Dr. Yung-Chang Chen and Dr. Chun-Chieh Huang (Department of Orthopaedic Surgery, National Taiwan University Hospital), Dr. Hsien-Te Peng (Chinese Culture University, Taipei, Taiwan), and the members of the Performance Analysis Laboratory (NTUPT) for supporting and helping with this study.

\section{CONFLICTS OF INTEREST}

Declared none.

\section{REFERENCES}

[1] McConnell J. Management of patellofemoral problems. Man Ther 1996; 1: 60-6.

[2] Almeida SA, Trone DW, Leone DM, Shaffer RA, Patheal SL, Long K. Gender difference in musculoskeletal injury rates: a function of symptom reporting? Med Sci Sports Exerc 1999; 31: 1807-12.

[3] Witvrouw E, Werner S, Mikkelsen C, Van Tiggelen D, Vanden Berghe L, Cerulli G. Clinical classification of patellofemoral pain syndrome: guidelines for non-operative treatment. Knee Surg Sports Traumatol Arthrosc 2005; 13: 122-30.
[4] Wilson T. The measurement of patellar alignment in patellofemoral pain syndrome: are we confusing assumptions with evidence? J Orthop Sports Phys Ther 2007; 37: 330-41.

[5] Elias DA, White LM. Imaging of patellofemoral disorders. Clin Radiol 2004; 59: 543-57.

[6] Draper CE, Besier TF, Santos JM, Jennings F, et al. Using realtime MRI to quantify altered joint kinematics in subjects with patellofemoral pain and to evaluate the effects of a patellar brace or sleeve on joint motion. J Orthop Res 2008; 27: 571-7.

[7] Laprade J, Culham E. Radiographic measures in subjects who are asymptomatic and subjects with patellofemoral pain syndrome. Clin Orthop Relat Res 2003; 414: 172-82.

[8] Lin YF, Lin JJ, Jan MH, Wei TC, Shih HY, Cheng CK. Role of the vastus medialis obliquus in repositioning the patella. A dynamic computed tomography study. Am J Sports Med 2008; 36: 741-7.

[9] MacIntyre NJ, Hill NA, Fellows RA, Ellis RE, Wilson DR. Patellofemoral joint kinematics in individuals with and without patellofemoral pain syndrome. J Bone Joint Surg 2006; 88A: 2596605 .

[10] Sheehan FT, Derasari A, Brindle TJ, Alter KE. Understanding patellofemoral pain with maltracking in the presence of joint laxity: complete 3D in vivo patellofemoral and tibiofemoral kinematics. J Orthop Res 2009; 27: 561-70.

[11] Nha KW, Papannagari R, Gill TJ, et al. In vivo patellar tracking: clinical motions and patellofemoral indices. J Orthop Res 2008; 26 : 1067-74.

[12] Varadarajan KM, Gill TJ, Freiberg AA, Rubash HE, Li G. Patellar tendon orientation and patellar tracking in male and female knees. J Orthop Res 2010; 28: 322-8.

[13] Laprade J, Lee R. Real-time measurement of patellofemoral kinematics in asymptomatic subjects. Knee 2005; 12: 63-72.

[14] Wilson NA, Press JM, Koh JL, Hendrix RW, Zhang LQ. In vivo noninvasive evaluation of abnormal patellar tracking during squatting in patients with patellofemoral pain. J Bone Joint Surg 2009; 91A: 558-66.

[15] Lin F, Makhsous M, Chang AH, Hendrix RW, Zhang LQ. In vivo and noninvasive six degrees of freedom patellar tracking during voluntary knee movement. Clin Biomech 2003; 18: 401-9.

[16] Cutti AG, Cappello A, Davalli A. In vivo validation of a new technique that compensates for soft tissue artifact in the upper-arm: preliminary results. Clin Biomech 2006; 21 (Suppl 1): S13-9.

[17] Portney LG, Watkins MP, Eds. Foundations of clinical research: applications to practice. New Jersey: Pearson Education Inc. 2000. 